\title{
THE PECULIARITIES OF CROSS-CORRELATION BETWEEN TWO SECONDARY PRECURSORS - RADON AND MAGNETIC FIELD VARIATIONS, INDUCED BY TECTONIC ACTIVITY
}

\author{
V.D. Rusov ${ }^{*}$, V.Yu. Maksymchuk ${ }^{2}$, R. Ilicí3,4, V.M. Pavlovych ${ }^{5}$, V.G. Bakhmutov', \\ D.N. Saranuk ${ }^{1}$, V.M. Vaschenko ${ }^{7,8}$, J. Skvarč ${ }^{4}$, L. Hanžič ${ }^{3}$, S.I. Kosenko ${ }^{1}$ \\ ${ }^{1}$ Odessa National Polytechnic University, Shevchenko av. 1, 65044 Odessa, Ukraine \\ ${ }^{2}$ Carpathian Branch of Institute of Geophysics, National Academy of Science, Naukova st. 3-b, 79053 Lviv, Ukraine \\ ${ }^{3}$ Faculty of Civil Engineering, University of Maribor, Smetanova 17, 2000 Maribor, Slovenia \\ ${ }^{4} J$. Stefan Institute, Jamova 39, 1000 Ljubljana, Slovenia \\ ${ }^{5}$ Institute for Nuclear Research, Pr. Nauki 47, 03028 Kiev, Ukraine \\ ${ }^{6}$ Institute of Geophysics, National Academy of Science, Palladina av. 32, 03680 Kiev, Ukraine \\ ${ }^{7}$ Ukrainian Antarctic Centre, Tarasa Schevchenko Blvd. 16, 01601 Kiev, Ukraine \\ ${ }^{8}$ National Taras Shevchenko University, Prosp. Glushkova 2, 03022 Kiev, Ukraine
}

\begin{abstract}
A model of precursor manifestation mechanisms, stimulated by tectonic activity and some peculiarities of observer strategy, whose main task is the effective measurement of precursors in the spatial area of their occurrence on the Earth's daylight, are considered. In particular, the applicability of Dobrovolsky's approximation is analyzed, when an unperturbed medium (characterized by the simple shear state) and the area of tectonic activity (local inhomogeneity caused by the change only of shear modulus) are linearly elastic, and perturbation, in particular, surface displacement is calculated as a difference of the solutions of two independent static problems of the theory of elasticity with the same boundary condition on the surface. Within the framework of this approximation a formula for the spatial distribution (of first component) of magnetic field variations caused by piezomagnetic effect in the case of perturbed regular medium, which is in simple shear state is derived. Cogent arguments in favor of linear dependence between the radon spatial distribution and conditional deformation are obtained.

Changes in magnetic field strength and radon concentrations were measured along a tectonomagnetic profile of the total length of 11 $\mathrm{km}$ in the surroundings of the "Academician Vernadsky" Station on the Antarctic Peninsula (W $64^{\circ} 16^{\prime}, \mathrm{S}^{6} 65^{\circ} 15^{\prime}$ ). Results showed a positive correlation between the annual surface radon concentration and annual changes of magnetic field relative to a base point, and also the good coincidence with theoretical calculation.
\end{abstract}

Key words: Antarctica, Radon, CR-39, Magnetic field, Tectonic activity.

\section{Introduction}

The information about the concentration of radon isotopes and radon daughters in the air and soil is actively used all over the world for geophysical purposes (Åkerblom and Mellander, 1997; Hakl et al., 1997; Fleischer et al., 1997; Monnin and Seidel 1997; Khan et al., 1977; Balcázar, 1997; Guera and Lombardi, 2001; Zmazek et al., 2003; Kharatain et al., 2002; Majudar, 2004). Monitoring the tectonic activity of the Earth's crust is performed on the basis of several geophysical and chemical methods, including determination of the radon concentration in soil gas and in underground water (e.g. Monnin and Siedel, 1991). The applicability of radon is based on the fact that the high temperature of aquifers or geothermal water sources, which are neighbors of natural breaks, promotes the transport of radon upward along existing and/or forming breaks (Segovia, 1991; Singh et al., 1991; Durrani and Ilić, 1997). Measurement of the radon

"Corresponding author: Prof. Rusov V.D., E-mail: siiis@te.net.ua concentration is widely used in seismic testing areas to study active tectonic breaks and earthquake precursors. It is considered that radon is removed by underground waters from cracks in the Earth's crust just made in deformation processes. The close correlation of changes of radon concentration with time in underground waters with movements of the Earth's crust before earthquakes is evidence of that. According to an analogous mechanism, the zones of active tectonic breaks are characterized by anomalous radon concentration.

Study of the tectonic activity in the region of the location of the "Academician Vernadsky" Antarctic station (W $64^{\circ} 16^{\prime}, \mathrm{S} 65^{\circ} 15^{\prime}$ ) is important because large and deep breaks were revealed near the station. Furthermore the recent eruptive activity on Deception Island and neovolcanic zone along of Bransfield Strait show the high geodynamic activity on the North of our region (Smellie, 1988). The distance between the southern earthquakes in Bransfield Strait rift propagation and Vernadsky station is about $230 \mathrm{~km}$.

Present geodynamic and seismic tectonic processes, in particular in break zones, lead to changes of mechanical, electrical, magnetic and other properties of rocks. The physical mechanisms of their influence on the variation of magnetic field are due to piezomagnetism and electrokinetic effects. As a result, the temporary changes of geomagnetic field occur with the periods from a few weeks to several years, and the amplitudes from 1 to several tens of nT. These geomagnetic field variations due to the piezomagnetic effect, which are produced by tectonomagnetic anomalies, are the indicators of active geodynamic processes (current movements of the Earth's crust, earthquakes, vulcanization, etc.). They can be revealed by repeated and precise magnetic measurements (Skovorodkin et al., 1985).

The present work has a twofold aim. First, it is a long-term study of local temporary changes of geomagnetic fields caused by different physical and chemical processes in the Earth's crust. Secondly, it is a search for a correlation 
between the radon concentration, which reflects the tectonic activity of the Earth's crust, and temporary changes of the abnormal magnetic field. Our results obtained so far are summarized in the present paper.

\section{Theory}

It is well known that the tectonic processes due to regular deformation of the Earth crust are the main cause of existent background deformation field.

We consider that unperturbed medium is at background deformation field, which is supported by corresponding (regional) tectonic processes. We also consider that there is the volume $\mathrm{V}$ of tectonic activity, in other words, nonregular deformation volume inside a large unperturbed medium limited by the surface $S_{=} S_{0}+S_{1}($ Fig. 1). This local volume has changed properties (heterogeneity), which causes the corresponding perturbations of different geophysical fields in the Earth crust. Traditionally we call such perturbations as "precursors". The main, or more exactly, primary precursor of tectonic activity is a so-called mechanical precursor, i.e. medium deformation, exceeding background deformation. All the other precursors including those discussed in this paper - anomalies of radon concentration and magnetic variations - are the secondary in respect of Earth crust deformations.

A model describing mechanisms of precursor manifestations stimulated by tectonic activity and some peculiarities of observation strategy for effective precursor measurements in spatial zone of their manifestation on the daylight of the Earth is presented below.

\subsection{The distributions of deformations and radon concentrations}

We define a zone of precursor activity manifestation as a part of daylight, which contains the epicenter of tectonic stress and is limited by a line where precursor perturbations are of the same magnitude as the background variations. Taking into account this definition let us consider the construction of deformation distribution on daylight for a typical case of tectonic activity. Note that the main idea of such problem solution (excluding some modification of process phenomenology) is completely based on Dobrovolsky theory of seismic focus evolution (Dobrovolsky, 1984).

As it stated above, the Earth crust moves with approximately constant (on regional scale) strain rate, which maintains a constant (in mentioned sense) level of shearing stress against a background of almost hydrostatical stress field due to gravitation.

A viscous-elastic medium is the simplest model of continuous medium, which reflects the stated properties. However the high effective viscosity of the Earth core (which is $10^{20}-10^{22} \mathrm{~Pa} / \mathrm{s}$ according to different estimates), relatively short duration of investigated processes (1 - 10 years) and necessity to study the difference of states rather than the characteristics of absolute state make it possible to introduce a following simplification. We consider an approximation, when the unperturbed medium and the volume of tectonic activity (local heterogeneity) are linearly elastic and the perturbation is calculated as the difference of the solutions of two separate statistical problems of theory of elasticity with the same boundary conditions on surface (Fig. 1). Equal boundary conditions stipulated by the fact that the energy of tectonic processes considerably exceeds the increment of the energy due to the formation of the area of increased tectonic activity and therefore the appearance of local heterogeneity cannot distort the regular tectonic processes far off its hypocenter.

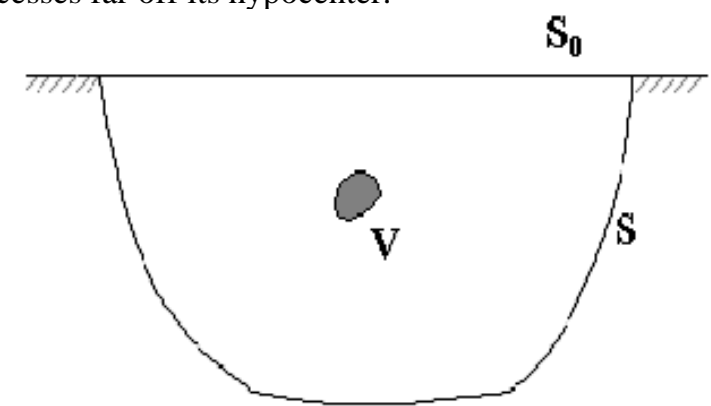

Fig. 1. The tectonic activity area $\mathrm{V}$ in medium.

Let us consider half-space $\mathrm{x}_{3} \geq 0$, which contains homogeneous and isotropic medium. The problem is set in rectangular coordinates $\mathrm{x}_{\mathrm{i}}$ (the comma of the lower index denotes differentiation by corresponding spatial coordinate and the recurring indexes cause summation). In the general case, at small $\alpha$, which characterize the weight of perturbed part of elastic modulus, the perturbed displacements $\mathrm{w}_{\mathrm{r}}$ of such medium are described by Dobrovolsky's formula (1984)

$$
w_{r}(x)=-\alpha\left[\frac{\sigma_{l l}^{0}}{3} \frac{K^{\prime}}{K^{0}} \delta_{i}^{j}+\frac{\mu^{\prime}}{\mu^{0}}\left(\sigma_{i j}^{0}-\frac{\sigma_{l l}^{0}}{3} \delta_{i}^{j}\right)\right] \iiint_{V} v_{i, j}^{r}(\xi, x) d v_{\xi},
$$

where $\sigma_{i j}^{0}=c_{i j k l}^{0} \varepsilon_{k l}^{0}$ is unperturbed stress equal to the product of unperturbed elastic modulus $c_{i j k l}^{0}$ and deformation value $\varepsilon_{k l}^{0}, \mathrm{~K}^{\prime}$ and $\mathrm{K}^{0}$ are the bulk elastic modules, $\mu^{\prime}$ and $\mu^{0}$ are shear modules in perturbed and unperturbed state, respectively, $\delta_{i}^{j}$ are Kronecker indexes, $v_{i}^{r}(\mathrm{x}, \xi)$ is Green function (tensor), $\xi$ are the coordinates of the origin of unit point force and $\mathrm{V}$ is the volume of the perturbed area of investigated medium. 
According to Dobrovolsky, we suppose that only shear modulus is changed in the area of increased tectonic activity $\mathrm{V}$, i.e. $\mathrm{K}^{\prime}=0, \mu^{\prime}=\mu^{0}=\mu$, and regular (unperturbed) state is state of pure shear with stress

$\sigma_{12}^{0}=\sigma_{21}^{0}=\tau$, for another $\sigma_{i j}^{0}=0$.

Then Eq. (2) with allowance of Eq. (3) has the following form

$$
w_{r}(x)=-\alpha r \iint_{V}\left[v_{1,2}^{r}(x, \xi)+v_{2,1}^{r}(x, \xi)\right] d v_{\xi} \text {. }
$$

At the same time, when surface displacements are calculated the Green function $v_{i}^{r}$ is the solution of the problem of point unit force applied on surface, i.e. so-called Businesk-Cherutti tensor (Novatsky, 1975). For the r-th unit power $\delta_{i}^{r}$ applied in the point $\left(\xi_{1}, \xi_{2}, 0\right)$ we have

$$
\begin{gathered}
v_{i}^{r}(x, \xi)=\frac{1}{4 \pi \mu}\left[\left(\frac{1}{R}+\frac{1-2 v}{R+x_{3}}\right) \delta_{i}^{r}+\left(\frac{1}{R}-\frac{1-2 v}{R\left(R+x_{3}\right)^{2}}\right)\left(x_{i}-\xi_{i}\right)\left(x_{r}-\xi_{r}\right)+\right. \\
\left.+\frac{1-2 v}{R\left(R+x_{3}\right)}\left(\left(x_{r}-\xi_{r}\right) \delta_{i}^{3}-\left(x_{i}-\xi_{i}\right) \delta_{r}^{3}\right)+\frac{(1-2 v) x_{3}}{R\left(R+x_{3}\right)^{2}}\left(\left(x_{r}-\xi_{r}\right) \delta_{i}^{3}+\left(x_{r}-\xi_{r}\right) \delta_{i}^{3}\right)++\left(\frac{1-2 v}{R}-\frac{1-2 v}{R+x_{3}}-\frac{x_{3}^{2}(1-2 v)}{R+x_{3}}\right) \delta_{i}^{3} \delta_{r}^{3}\right],
\end{gathered}
$$

where $v$ is Poisson's ratio, and

$$
R=\sqrt{\left(\left(x_{1}-\xi_{1}\right)^{2}+\left(x_{2}-\xi_{2}\right)^{2}+x_{3}^{2}\right)} .
$$

It turns out that the perturbed displacements, Eq. (4), with allowance of Eq. (5) can be calculated in elementary functions in case, when area V is a parallelepiped (Dobrovolsky and Mjachkin, 1976). It is also shown ibidem that for areas having more complex form or for points located outside of area $\mathrm{V}$, sufficiently far from this area, it is convenient to calculate $\mathrm{w}_{\mathrm{r}}$ approximately, substituting the integrand by its value in the some internal point of volume, which coincides with the center of area $\mathrm{V}$, when it is symmetric (monopole approximation). Note that the error of such approximate calculation is less than a few percents even in epicentre zone in comparison with exact solution (Dobrovolsky and Mjachkin, 1976).

Let us place the centre of area $\mathrm{V}$ at point $(0,0, \mathrm{H})$ and introduce the following designations

$x=x_{1}, \quad y=x_{2}, \quad r=\sqrt{x^{2}+y^{2}+H^{2}}$.

Then the approximate calculation of integral in Eq. (4) gives displacements in the following form

$$
\begin{aligned}
& w_{1}=-\frac{\alpha V \tau}{2 \pi \mu} \frac{y}{r^{2}}\left[\frac{3 x^{2}}{r^{2}}+\frac{1-2 v}{(r+H)^{2}}\left(\frac{r^{2}-x^{2}}{r}-\frac{2 x^{2}}{r+H}\right)\right], \\
& w_{2}=-\frac{\alpha V \tau}{2 \pi \mu} \frac{x}{r^{2}}\left[\frac{3 y^{2}}{r^{2}}+\frac{1-2 v}{(r+H)^{2}}\left(\frac{r^{2}-y^{2}}{r}-\frac{2 y^{2}}{r+H}\right)\right], \\
& w_{3}=-\frac{\alpha V \tau}{2 \pi \mu} \frac{x y}{r^{2}}\left[\frac{3 H}{r^{2}}+\frac{(1-2 v)(2 r+H)}{(r+H)^{2}}\right] .
\end{aligned}
$$

Further, using Eqs. (8) - (10) the deformation tensor components are calculated

$$
\begin{aligned}
& \varepsilon_{x x}=\frac{\alpha V \tau}{2 \pi \mu} \frac{3 x y}{r^{3}}\left[\frac{2}{r^{2}}-\frac{5 x^{2}}{r^{4}}+\frac{1-2 v}{(r+H)^{3}}\left(\frac{2 x^{2}}{r+H}-\frac{\left(y^{2}+H^{2}\right)(3 r+H)}{r^{2}}\right)\right], \\
& \varepsilon_{y y}=\frac{\alpha V \tau}{2 \pi \mu} \frac{3 x y}{r^{3}}\left[\frac{2}{r^{2}}-\frac{5 y^{2}}{r^{4}}+\frac{1-2 v}{(r+H)^{3}}\left(\frac{2 y^{2}}{r+H}-\frac{\left(x^{2}+H^{2}\right)(3 r+H)}{r^{2}}\right)\right], \\
& \varepsilon_{x y}=\frac{\alpha V \tau}{2 \pi \mu} \frac{1}{r^{3}}\left[\frac{3\left(r^{2}-H^{2}\right)}{2 r^{2}}-\frac{15 x^{2} y^{2}}{r^{4}}+\frac{1-2 v}{2(r+H)^{2}} \times\right. \\
& \left.\times\left(6 r^{2}-4 H^{2}+\frac{4 x^{2} y^{2}(5 r+2 H)}{r(r+H)^{2}}-\frac{\left(r^{4}+x^{4}+y^{4}-H^{4}\right)(5 r+3 H}{r^{2}(r+H)}\right)\right] .
\end{aligned}
$$

In order to present the strain distribution on daylight in a convenient or universal form we introduce the nondimensional variables

$$
\xi=x / \rho, \quad \eta=y / \rho, \quad h=H / \rho
$$

and conditional deformation

$$
\omega_{i j}=\frac{3 \mu}{2 \alpha \tau} \varepsilon_{i j},
$$

where $\rho$ is the average radius of area $V$, i.e. the sphere radius of same volume.

\subsection{The distributions of deformations and magnetic field}

It is well known that variation of mechanical stress field causes corresponding magnetization variation $\Delta \mathrm{I}$ in the every point of solid body. In other words, the piezomagnetic effect is explained by the variation of magnetic induction, induced by the change of elementary magnetic dipole, due to the variation of the magnetic moment of every volume element. The total effect may be calculated by integration by full volume. 
Note that systematic study of this phenomenon showed that under conditions of increased tectonic activity (including processes of earthquake preparation) the magnetic field variations are appeared. These variations are really determined by the above-mentioned processes and may be caused by piezomagnetic effect (Breiner, 1964; Skovorodkin, 1985; Maksimchuk et al., 2003). Moreover the expression for piezomagnetism describing was obtained by Johnston (1973), but it is represented in co-ordinates coinciding with principal axes of stress tensor, i.e.

$$
\Delta I_{i}=C I_{i}\left(\frac{\sigma_{j}+\sigma_{k}}{2}-\sigma_{i}\right),
$$

where $\sigma_{\mathrm{i}}, \sigma_{\mathrm{j}}$ and $\sigma_{\mathrm{k}}$ are principal stresses (all $\mathrm{i}, \mathrm{j}$ and $\mathrm{k}$ are different) and $\mathrm{C}$ is piezomagnetic coefficient.

Though all terms in Eq. (16) are tensors, the equation is not of invariant (relative to rotation) tensor form. However, as Dobrovolsky (1984) showed that Eq. (16) may be written in a more handy form. Really, there is an obvious equality

$$
\frac{\sigma_{j}+\sigma_{k}}{2}-\sigma_{i}=-\frac{3}{2}\left(\sigma_{i}-\frac{\sigma_{i}+\sigma_{j}+\sigma_{k}}{3}\right) .
$$

Here the expression in brackets is the deviator of stress tensor in principal axes. If deviator components are expressed as $\mathrm{s}_{\mathrm{ij}}$, the Eq. (16) has the following form

$$
\Delta I_{i}=-\frac{3}{2} C I_{j} s_{i j}
$$

where there is summation by recurring indexes. It is obvious that, by virtue of rules of tensor algebra, Eq. (18) in such form is invariant relative to rotations and therefore is valid in arbitrary co-ordinates.

Now let us calculate the distribution of magnetic field regional variations $\Delta \mathrm{F}_{\mathrm{r}}$ determined by difference between the total vector of magnetic induction and the background induction $\vec{B}_{E}$, characterized exceptionally by basic terrestrial magnetic field

$$
\Delta B_{r}=\left|\vec{B}_{E}+\Delta \vec{B}_{r}\right|-\left|\vec{B}_{E}\right|
$$

When $\left(\left(\Delta \vec{B}_{r}\right)^{2} / B_{E}\right)<<1$ the Eq. (19), with adequate accuracy, may be presented as

$$
\Delta B_{r}=\frac{\vec{B}_{E}}{B_{E}} \Delta \vec{B}_{r}=\vec{b} \cdot \Delta \vec{B}_{r},
$$

where $\vec{b}$ is unit vector of basic terrestrial magnetic field.

On the other hand, it is well known that magnetic induction may be unambiguously expressed by vector potential $\vec{A}$ in the following way

$$
\vec{B}=\operatorname{rot} \vec{A}, \text { if } \operatorname{div} \vec{A}=0,
$$

where the equality to zero of vector potential divergence ensures the potential calibration.

From magnetism physics it is also known that vector potential of magnetic dipole with magnetic moment $\vec{m}$ has form

$$
\vec{A}=k \frac{\vec{m} \times \vec{R}}{R^{3}},
$$

where $\mathrm{k}$ is the coefficient dependent on used system of units, and $\vec{R}$ is the radius vector directed from dipole point to the point of observation.

If in volume element $\mathrm{dv}$ the change of magnetization $\Delta \vec{I}$ was occurred, it would cause magnetic momentum variation of value $d \vec{m}=\Delta \vec{I} d v$ and the corresponding variation of vector potential

$$
d \vec{A}=k \frac{\Delta \vec{I} \times \vec{R}}{R^{3}} d v
$$

Integration of the Eq. (23) over the total Earth's core layer from daylight to Curie isotherm gives the total variation of vector potential. It reduces to the following form of Eq. (21)

$$
\Delta \vec{B}=k \cdot \operatorname{rot} \iiint \frac{\Delta \vec{I} \times \vec{R}}{R^{3}} d v=k \nabla \times \iiint \frac{\Delta \vec{I} \times \vec{R}}{R^{3}} d v,
$$

where $\nabla$ is nabla operator.

The insertion of expression for magnetization, Eq. (18) in Eq. (24), taking into account scalar product (Eq. (20)) and considering also the Levi-Civita symbols $\mathrm{e}_{\mathrm{ijk}}$, the final solution for $\Delta \mathrm{B}$ has the form

$$
\Delta B=-\frac{3}{2} k C b_{p} e_{m k p} \nabla_{m} \iiint \frac{e_{i j k} I_{l} s_{i l}^{\prime} R_{j}}{R^{3}} d v_{\xi},
$$

where $\nabla_{\mathrm{m}}=\partial / \partial \mathrm{x}_{\mathrm{m}} ; \mathrm{R}_{\mathrm{j}}=\mathrm{x}_{\mathrm{j}}-\xi_{\mathrm{j}}\left(\mathrm{x}_{\mathrm{j}}\right.$ is the coordinates of the point of observation, $\xi_{\mathrm{j}}$ is the integration variable) and $s_{i l}^{\prime}$ is the stress tensor deviator.

Further we shall use the expression of stress increment obtained in (Dobrovolsky, 1984)

$$
\sigma_{i j}^{\prime}=\alpha \sigma_{i j}^{0} \delta_{V}+\sigma_{i j}^{\prime \prime} \text {, }
$$


where $\sigma_{i j}^{\prime \prime}=c_{i j k l}^{0} w_{k l}\left(c_{i j k l}^{0}\right.$ is the elastic modulus of unperturbed medium $), \mathrm{w}_{\mathrm{kl}}=\mathrm{u}_{\mathrm{kl}}-u_{k l}^{0}$, i.e. the difference of perturbed and unperturbed displacements and $\delta_{\mathrm{V}}$ is the characteristic function of volume V. In this case Eq. (26) in corresponding deviators looks like

$$
\begin{aligned}
& s_{i j}^{\prime}=\alpha s_{i j}^{0} \delta_{V}+s_{i j}^{\prime \prime} . \\
& \text { It follows from Eq. (27) that Eq. (25) divides into two parts } \\
& \Delta B_{r}=\Delta B_{r 1}+\Delta B_{r 2} .
\end{aligned}
$$

Let us investigate both components separately. In the first item,

$$
\Delta B_{r 1}=-\frac{3}{2} k C b_{p} e_{m k p} \nabla_{m} \iiint \frac{e_{i j k} I_{l} \alpha s_{i l}^{o} R_{j}}{R^{3}} d v,
$$

the integration has place only by the volume of area $\mathrm{V}$, therefore the assumption $\vec{I}=$ const is appropriate for the estimation. Since it is possible to consider that $\vec{I} \| \vec{B}_{E}$ for the isotropic area V, we have $\mathrm{I}_{1}=\mathrm{Ib}_{1}$. As a result, Eq. (29) with allowance for $s_{i j}^{0}=$ const takes form

$$
\Delta F_{r 1}=-\frac{3}{2} k C \alpha I b_{p} e_{m k p} \nabla_{m} e_{i j k} b_{l} s_{i l}^{0} \iiint \frac{R_{j}}{R^{3}} d v .
$$

As far as we are interested in distribution of magnetic field variations on the Earth's daylight, calculating integral in Eq. (30) in monopole approximation, we obtain an approximation for Eq. (30)

$$
\Delta F_{r 1}=-\frac{3}{2} k C \alpha I b_{p} e_{m k p} \nabla_{m} e_{i j k} b_{l} s_{i l}^{0} \frac{r_{j}}{r^{3}}
$$

where $\mathrm{r}_{\mathrm{j}}=\mathrm{x}_{\mathrm{j}}-\xi_{j}^{\prime}$ and $\xi_{j}^{\prime}$ are coordinates of some point within area $\mathrm{V}$.

On the basis of exact calculation of Eq. (30) for a sphere, which tangents to half-space boundary at the point of contact $\mathrm{x}_{\mathrm{j}}$, Dobrovolsky (1984) showed that monopole approximation (31) is satisfactory, if $\xi_{j}^{\prime}$ is located closer to daylight approximately on half radius relative to the center of area V. As a result we take in Eq. (31)

$$
\xi_{j}^{\prime}=\xi_{j}^{0}-\frac{1}{2} \delta_{3 j} \rho
$$

where $\xi_{j}^{0}$ are the coordinates of area $\mathrm{V}$ center and $\rho$ is the average radius of area $\mathrm{V}$, i.e. the radius of a sphere with equal volume.

The use of Eq. (32) is justified only for calculations in epicentre zone, whereas the admission $\xi_{j}^{\prime}=\xi_{j}^{0}$ already on the boundary gives the result differing from those calculated by Eq. (32) up to $25 \%$.

Since

$$
\nabla_{m} \frac{r_{j}}{r^{3}}=-\frac{1}{r^{3}}\left[\frac{3\left(x_{j}-\xi_{j}\right)\left(x_{m}-\xi_{m}\right)}{r^{2}}-\delta_{m}^{j}\right]=-\frac{1}{r^{3}} q_{j m},
$$

(where $\mathrm{q}_{\mathrm{jm}}=\mathrm{q}_{\mathrm{mj}}$ and $\mathrm{q}_{\mathrm{jj}}=0$ ) Eq. (31) after simplifications takes the form

$$
\Delta F_{r 1}=-\frac{3}{2} \frac{k C V \alpha I}{r^{3}} b_{m} b_{j} s_{i j}^{0} q_{i m} .
$$

As in our case a regular (unperturbed state) is considered as simple shear state with stress Eq. (3) we obtain from Eq. (34) and, consequently, from Eq. (29)

$$
\Delta F_{r 1}=-\frac{3}{2} \frac{k C V \alpha I}{r^{3}}\left[\left(b_{1}^{2}+b_{2}^{2}\right) q_{12}+b_{2} b_{3} q_{13}+b_{1} b_{3} q_{23}\right],
$$

where $b_{1}=\sin \theta_{1}$ and $b_{2}=\cos \theta_{2}, b_{3}=\sin \theta_{2}$. Here $\theta_{1}$ and $\theta_{2}$ are the angles determining the inclination and declination of the vector of the main terrestrial magnetic field in given region).

Thus, the Eq. (35) is improved Dobrovolsky (1984) formula for the spatial distribution of magnetic field variations (first component) in the case of perturbation of regular medium, which is the simple shear state. Here the multiplicative coefficient before the bracket contains the information about the scalar magnitudes of magnetic characteristics, stresses in medium and dependence on distance. The expression in the brackets, in general, describes the direction distribution and therefore is called direction function. The further concretisation of Eq. (35) for $\Delta \mathrm{F}_{\mathrm{r} 1}$ connected with magnetic description of the investigated Earth's region and the orientation of tectonic stresses are considered in the section 3.

\section{Experimental ${ }^{1}$}

The tectonomagnetic investigations in the vicinity of the "Academician Vernadsky" station began in 1998 along the sublatitudinal profile Barchans Islands - Rasmussen point (Fig. 2). In 2001 the first data about geomagnetic field $\Delta \mathrm{F}$ time changes were reported in (Maksymchuk et al., 2002). The next cycle of observations was carried out in 2002 and extended by addition of new tectonomagnetic sites of observation. The fourth, fifth and sixth measurements were performed in 2003, 2004 and 2005, respectively (Maksymchuk et al., 2004, 2006). Detailed results of these investigation will be published elsewere (Maksymchuk et al., 2006).

\footnotetext{
${ }^{1}$ The experiments were carried out during the 7th, 8th, 9th and 10th Ukrainian Antarctic Expeditions in March 2002, March 2003, March 2004 and March 2005, respectively.
} 


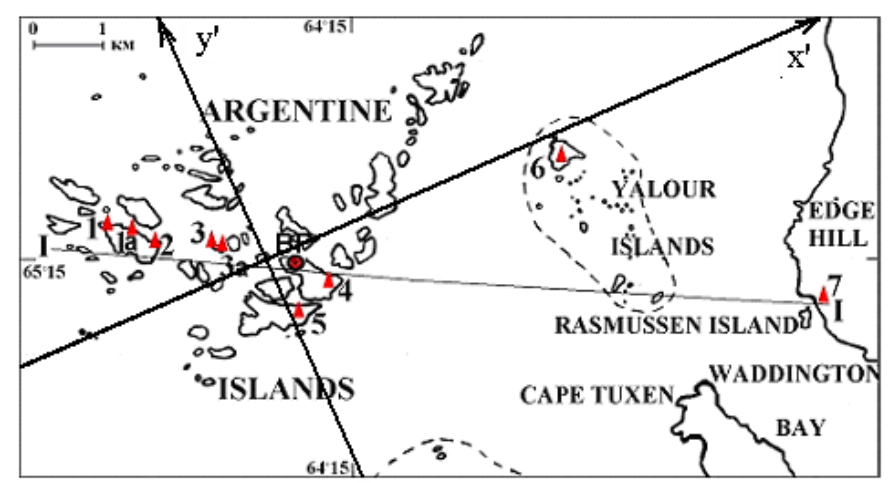

Fig. 2. Locations of geomagnetic and radon measurements in the region "Academician Vernadsky" Antarctic Station. BP - base points for magnetic and radon observation "Argentine Islands"; I - I - latitudianal profile. Axis X is line of maximal tangential stresses in given region.

The measurement of radon concentrations was performed at several sites close to the sites of tectonomagnetic observations along the Barchans-Rasmussen profile. These measurements was started during the 7th season Ukrainian Antarctic Expedition in 2002. It should be mentioned that in 2003 the Rn laboratory was set up close to the base point (BP), where continuous Rn measurements with an active Rn monitor were performed (Ilić et al., 2005). In 2004 an improved Rn monitoring procedure for etched track detectors was introduced and 3 additional continuous Rn devices were set up to extend Rn studies to other topics (Rusov et al., 2006).

\subsection{Site}

The" Academician Vernadsky" Antarctic station (the former UK station "Faraday") is located on Galindez Island (Archipelago of the Argentine Islands), part of the West Pacific Shelf of the Antarctic Peninsula (fig.2). The Antarctic Peninsula with its adjacent Islands forms a part of the West Antarctic folded system, which extends along the Pacific Coast of Western Antarctica. The origin of the Antarctic Peninsula is located in the southern part of the jointing zone of two large-scale geological structures - the Pacific and Gondvana segments - of the Earth. It is a result of long-time interaction of tectonic processes, which are peculiar to each of these planetary segments and cause their main peculiarities as a whole. The deep trench of the Wedell Sea and its outlying western and southwestern shelf belong to the Gondvana segment. At the present stage of evolution, the deep ocean trench and marine shelf area, as well as the Antarctic Peninsula with its adjacent islands and lands, belong to the Pacific Segment.

The evidence of the geodynamic activity of the region is neovolcanic zones and seismic activity in Bransfield Strait. The South Shetland Trench, which lies to N-W part of the South Shetland Islands, is the sole remnant zone of the subduction zone that existed along the entire Pacific margin of West Antarctica during the Mesozoic and Cenozoic [Baker, 1982]. Most of the subduction zone was progressively eliminated as the Phoenix-Antarctic spreading center was subducted beneath the Antarctic Peninsula. Slow subduction of the former Phoenix Plate appears to continue today within what is essentially a single Antarctic plate, owing to slab-pull forces and/or trench rollback The seismic, multibeam bathymetry and geodetic surveys data indicates that Bransfield Strait is undergoing active extension with the rate of $\sim 10 \mathrm{~mm} / \mathrm{yr}$ in direction orthogonal to the strait's strike [Chisteson et al., 2003]. The modern eruptive activity observed on Deception Island extends along this neovolcanic zone.

The most part of the western Antarctic Peninsula is represented by Meso-Cenozoic intrusive complex and JurassicCretaceous calk-alkaline AP Volcanic Group. The oldest rocks in Argentine Islands Archipelago are laves and pyroclastic members of the Upper Jurassic Volcanic Group which have been intruded by pre-Andian dykes and sills. The Andean Intrusive Suite has metamorphosed and metasomatized the volcanic and hypabyssal rocks. There is a later dyke phase which cuts both of volcanic and plutonic rocks. Finally, there are a few late comparatively unaltered Tertiary dykes. Whereas the Argentine Islands are composed entirely of the volcanic rocks (andesite laves and dacite pyroclastic rocks) the western part of archipelago occur the Andean Intrusive Suite rocks represented by granodiorites.

The tectonomagnetic points of observation were selected mainly in the Argentine Islands Archipelago (Fig. 2, Table 1). Two tectonomagnetic profiles could be chosen here: a latitudinal profile (Barchans Islands - Rasmussen point), and a meridian profile (Berselot Island - Booth Island). The total length of latitudinal profile is $11 \mathrm{~km}$ (having 7 observation points, denoted by $\mathrm{I}-\mathrm{I}$ in Fig. 2). The profile from $\mathrm{W}$ to E crosses the volcanic and plutonic rocks with the probably fault zone between them, the fault between Argentine Islands Archipelago and Antarctic Peninsula in Penola Strait which well traced in bathymetry and reaches the Antarctic Peninsula on the East.

Table 1.

Sites of measurements

\begin{tabular}{|c|c|c|}
\hline $\begin{array}{c}\text { Location } \\
\text { Number (n) }\end{array}$ & Location Name & Coordinates \\
\hline 1 & $\begin{array}{l}\text { Barhans Islands } \\
\text { west }\end{array}$ & 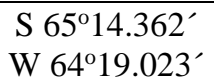 \\
\hline
\end{tabular}




\begin{tabular}{|l|l|c|}
\hline $1 \mathrm{a}$ & $\begin{array}{l}\text { Barhans Islands } \\
\text { central }\end{array}$ & $\begin{array}{c}\mathrm{S} 65^{\circ} 14.410^{\prime} \\
\mathrm{W} 64^{\circ} 18.447^{\prime}\end{array}$ \\
\hline 2 & $\begin{array}{l}\text { Barhans Islands } \\
\text { east }\end{array}$ & $\mathrm{S} 65^{\circ} 14.544^{\prime}$ \\
$\mathrm{W} 64^{\circ} 17.862^{\prime}$
\end{tabular}

\subsection{Monitoring of tectonomagnetic anomalies}

Tectonomagnetic anomalies, defined as abnormal temporary changes of geomagnetic field due to physical and chemical phenomena in the Earth's crust and upper mantle, are results of changes of seismic, volcanic and other geodynamic processes. The study of such changes of magnetic field is connected with some difficulties since their amplitude does not exceed several tens of $\mathrm{nT}$ and the spectrum overlaps with the spectrum of variations of internal (with the duration over 11 years) and external origin (ionosphere and magnetosphere with a duration from seconds to several years).

Among numerous physical mechanisms which can lead to the appearance of tectonomagnetic anomalies, piezomagnetic and electrokinetic effects are the most predominant. Both of these effects are connected with changes in the stressed and deformed state of the geological medium, which lead to changes of magnetic properties of the rocks and variation of the electrical current.

The piezomagnetic effect is manifested by a change of the magnitude and space distribution of the magnetization vector due to mechanical loading in the range of elastic deformation. Electrokinetic phenomena appear in an electrically neutral system as a whole and consist of the displacement of one phase (liquid or solid) by another under the influence of an external electric field, or in the appearance of an electrical current due to phase displacement under mechanical stress.

Theoretical calculation of the anomalous effects for both mechnisms gave approximate estimates, which showed that the changes of magnetic field $\Delta \mathrm{F}$ can vary in the range from 0.1 to $10 \mathrm{nT}$ with pressure variations of $1-100$ bars/year (Maksymchuk et al., 2001). Besides this, the anomalous effects strongly depend on the depth and shape of the heterogeneities, element contents, magnetic and electric properties, etc. It should be noted that the pressure, even in strong earthquakes, does not exceed 100 bar.

The classical method for detection of tectonomagnetic anomalies is based on measurements Earth's magnetic field at a constant set of the observation points in definite subsequent time intervals. Usually, for technical reasons, the absolute value of the Earth's magnetic field vector F is measured. In order to avoid the influence of external field changes, the work was performed according to the scheme of synchronous differential measurements of the field $\mathrm{F}_{\mathrm{BP}}$ at a base point (BP) and the field $F_{n}$ at given ordinary point (n) of observation. The field difference $\Delta F=F_{n}-F_{B P}$ is independent of the external field influence for small distances $(10-30 \mathrm{~km})$ between the base and ordinary points. Thus the parameter sought is $\Delta \Delta \mathrm{F}$ i.e. the change of $\Delta \mathrm{F}$ during the period between the cycles of observations defined as

$\Delta \Delta \mathrm{F}=\Delta \mathrm{F}_{\mathrm{Y}}-\Delta \mathrm{F}_{\mathrm{Y}-1}$

where $\Delta \mathrm{F}_{\mathrm{Y}}$ and $\Delta \mathrm{F}_{\mathrm{Y}-1}$ are the measured values of $\Delta \mathrm{F}$ in the year $\mathrm{Y}$ and $\mathrm{Y}-1$ respectively.

The interval between the cycles of measurements is determined by the problem under study and the parameters of the phenomena, and can amount to days, months or years. Taking into account the climatic conditions of Antarctica (Archipelago of the Argentine Islands), it is most convinient to make measurements once a year, i.e. the studied tectonomagnetic effects have a period of about one year.

As the base point of observation we used the Argentine Islands (AIA) magnetic observatory, where F was recorded by MB-01 proton magnetometer with a sensitivity of $\pm 0.1 \mathrm{nT}$. Measurements of the field at the set of ordinary points were performed by MPP-203 proton magnetometer. The standard deviation of the field determination was $0.5-1 \mathrm{nT}$. Results of geomagnetic field measurements are summarized in Table 2.

The geomagnetic observations offered information on the structure of the anomalous magnetic field $\Delta \mathrm{F}$ along the profile I $-\mathrm{I}$, and also its time changes - tectonomagnetic anomalies $-\Delta \Delta \mathrm{F}$. It was found that the $\Delta \mathrm{F}$ is strongly dependent on position (Table 2). Due to discrepancy of the magnetic properties of volcanic and intrusive rocks the differences of magnetic anomaly were more then $1000 \mathrm{nT}$ with the maximum in the eastern part of the profile, at Rasmussen Cape (point 7). In the western part of the profile, on Three Little Pigs Islands (point 3), a local anomaly $\Delta \mathrm{F}$ of intensity $600 \mathrm{nT}$ was detected also. Dynamic changes of magnetic field $\Delta \Delta \mathrm{F}_{2003-02}$ during the period $2002-2003$ 
(Table 2) varied from -1.9 (Barchans Islands) to $+4.2 \mathrm{nT}$ (Three Little Pigs Islands). The definite regularity of their distribution along the profile was noticeable despite the small value of $\Delta \Delta \mathrm{F}$. During the periods 2003-2004 Barchans Islands and 2004-2005 these values amounts -6.3 nT (Three Little Pigs Islands) to +3.3 nT (Barchans Islands) and -4. 9 nT (Three Little Pigs Islands) to +6.9 nT (Rasmusen Cape). The repeated observation of 2003, 2004, and 2005 confirms, in general, the stability of $\Delta \Delta \mathrm{F}$ distribution along the profile I - I observed in 2002 (Table 2). Similar anomalous effects, but with somewhat smaller amplitude, are the general characteristic of active breaks in the Earth's crust and were revealed in many seismoactive zones of the world (Maksymchuk et al., 2002).

\subsection{Radon monitoring}

Long term measurements of radon concentration where carried out by passive radon dosimeter utilizing CR-39 and Makrofol E detectors. For this purpose the radon monitoring devices of the J. Stefan Institute (IJS), Ljubljana (Šutej et al., 1986) and Nuclear Center Karlsruhe (KfK), Karlsruhe (Urban and Piesch, 1981) were used. The dosimeters were placed on the Earth's surface (with the opening of the dosimeter, covered by a filter, looking downwards) and/or about $40 \mathrm{~cm}$ above the Earth surface (the dosimeters were fixed on styropore support). The measurements performed in $2002-$ 2003 by IJS dosimeter is described in (Ilić et al., 2005). Due to technical problems all of the measurements in 2003 2004 unfortunately were lost. At each sites at least 7 dosimeters were positioned to get information on the reproducibility of the results. The dosimeters were covered by a carbon steel oil drum $(80 \mathrm{~cm}$ dia and $1 \mathrm{~m}$ in height, used for $200 \mathrm{~L}$ volume). The detectors were exposed about one year. Before and/or after the exposure the detector foils were placed in aluminized foil to eliminate Rn exposure during transport to/from Europe/Antarctica. The CR-39 foils were etched in $6.25 \mathrm{~N} \mathrm{NaOH}$ at $70^{\circ} \mathrm{C}$ for 8 hours, and evaluated by the TRACOS system (Skvarč and Golovchenko, 2001). The response of the dosimeter to ${ }^{222} \mathrm{Rn}$ is 0.15 (tracks $\left.\mathrm{cm}^{-2}\right) /\left(\mathrm{Bq} \mathrm{m}^{-3}\right.$ day). The background track density was about 100 tracks $/ \mathrm{cm}^{2}$. Etching and reading of KfK dosimeteres were performed in Karlsruhe. It should be mentioned that due to moisture collections KfK dosimeters are not able to measure the radon concentration at the surface of the Earth. Average results of radon concentration measurements are given in Table 2.

\section{Results}

The isolines of conditional deformation $\omega_{\mathrm{xx}}$ calculated by Eqs. (11) and (15) at Poisson's ratio $v=0.25$ and the average depth of local heterogeneity occurrence $h=1,2,4$ are shown in Fig. 3. Note that sympol $h=H / \rho(14)$. From Fig. 3 it is possible to see that the strain distribution is strongly irregular. For deformation $\omega_{\mathrm{xx}}$ the coordinate axes, which coincide with the direction of maximal tangent stress in given region, are zero isolines and moreover there is an additional zero isoline in every quadrant. Thus, it is possible to miss the signal even quite close to the epicenter of tectonic activity, if the observation point is poorly chosen.

Experimental results of the annual radon concentration vs. change of difference of magnetic field for 2004-2005 are shown in Fig. 4. Comparison of the radon concentration values and the tectonomagnetic results shows a clear correlation.

The following function was fitted to the experimental data (Fig. 4).

$\mathrm{C}_{\mathrm{n}}=\mathrm{C}_{\mathrm{BP}}+\mathrm{C}_{\infty}\left[1-\exp \left(-\left|\Delta \Delta \mathrm{F}_{\mathrm{n}}+\Delta \Delta \mathrm{F}_{\mathrm{d}}\right|^{2} / \square \square \Delta \Delta \mathrm{F}_{\mathrm{o}}\right)^{2}\right]$

where $\mathrm{C}_{\infty}$ is the maximal radon concentration for a given area and $\Delta \Delta \mathrm{F}_{\mathrm{o}}$ is the change of the magnetic field, which corresponds to destructive (failure) stress, and $\Delta \Delta \mathrm{F}_{\mathrm{d}}$ is a constant describing displacement of the base point. It was found that $\Delta \Delta \mathrm{F}_{\mathrm{o}}=3.78 \mathrm{nT}, \mathrm{C}_{\mathrm{BP}}=3.3 \mathrm{~Bq} / \mathrm{m}^{3}, \mathrm{C}_{\infty}=5 \mathrm{~Bq} / \mathrm{m}^{3}$ and $\Delta \Delta \mathrm{F}_{\mathrm{d}}=1.2 \mathrm{nT}$. Note when $\left|\Delta \Delta \mathrm{F}_{\mathrm{n}}\right|<<\Delta \Delta \mathrm{F}_{\mathrm{o}}$, and $\Delta \Delta \mathrm{F}_{\mathrm{d}}=$ 0 , the Eq. (37) is transformed to

$\mathrm{C}_{\mathrm{n}}=\mathrm{C}_{\mathrm{BP}}+\mathrm{c}\left|\Delta \Delta \mathrm{F}_{\mathrm{n}}\right|$

where $\mathrm{c}=\mathrm{C}_{\infty} / \Delta \Delta \mathrm{F}_{\mathrm{o}}$ was estimated to be of the order of magnitude $1 \mathrm{~Bq} \mathrm{~m}^{-3} / \mathrm{nT}$. 

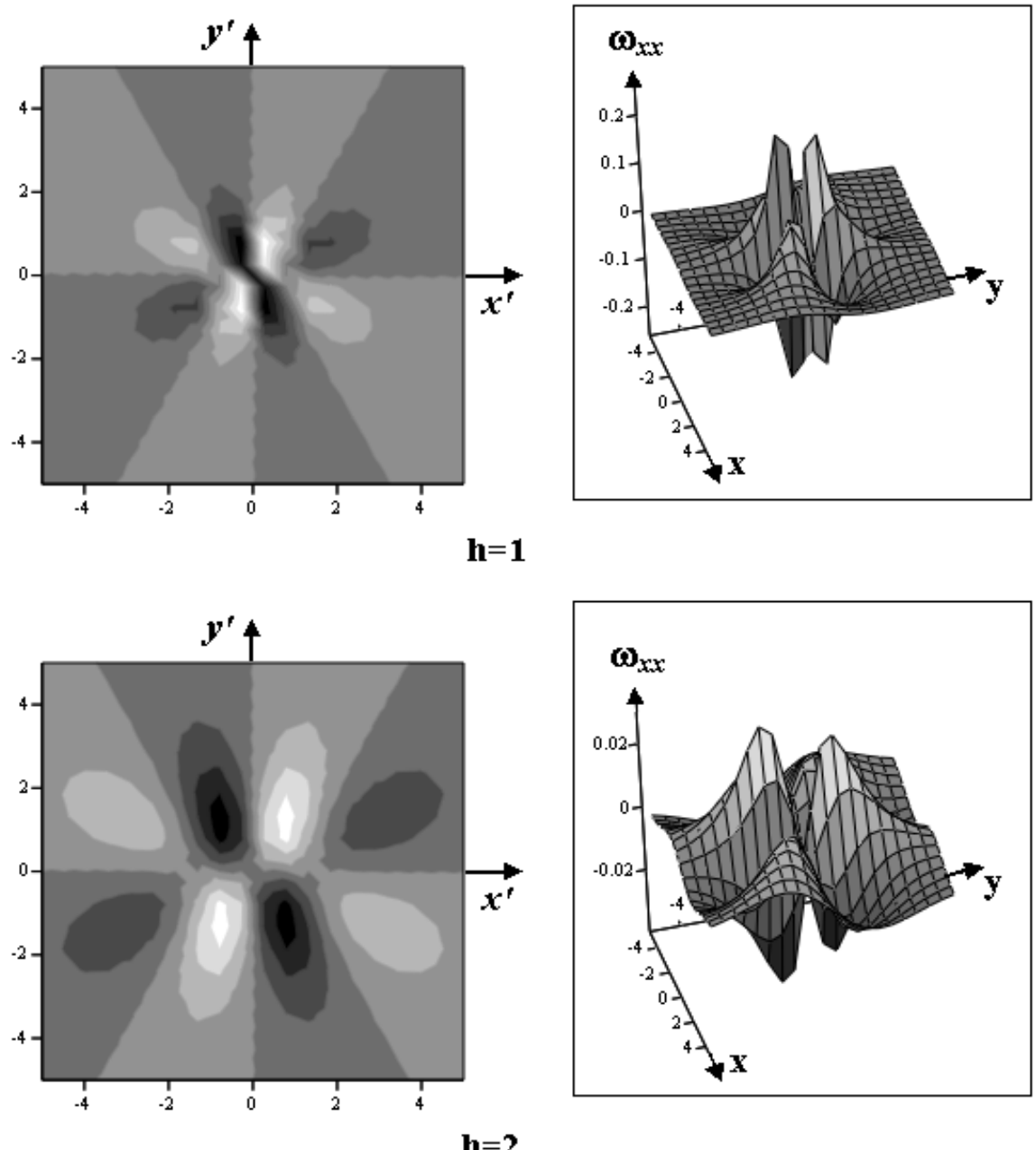

$\mathbf{h}=\mathbf{2}$
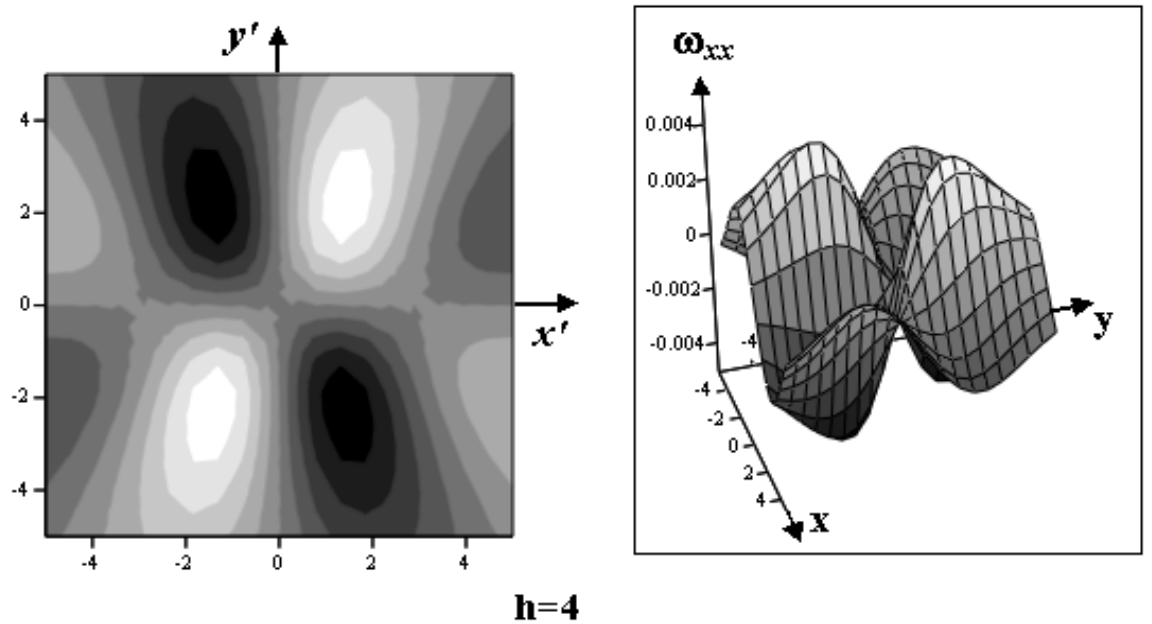

Fig. 3. The distribution of conditional deformation $\omega_{\mathrm{xx}}$ at different value of $h$. 


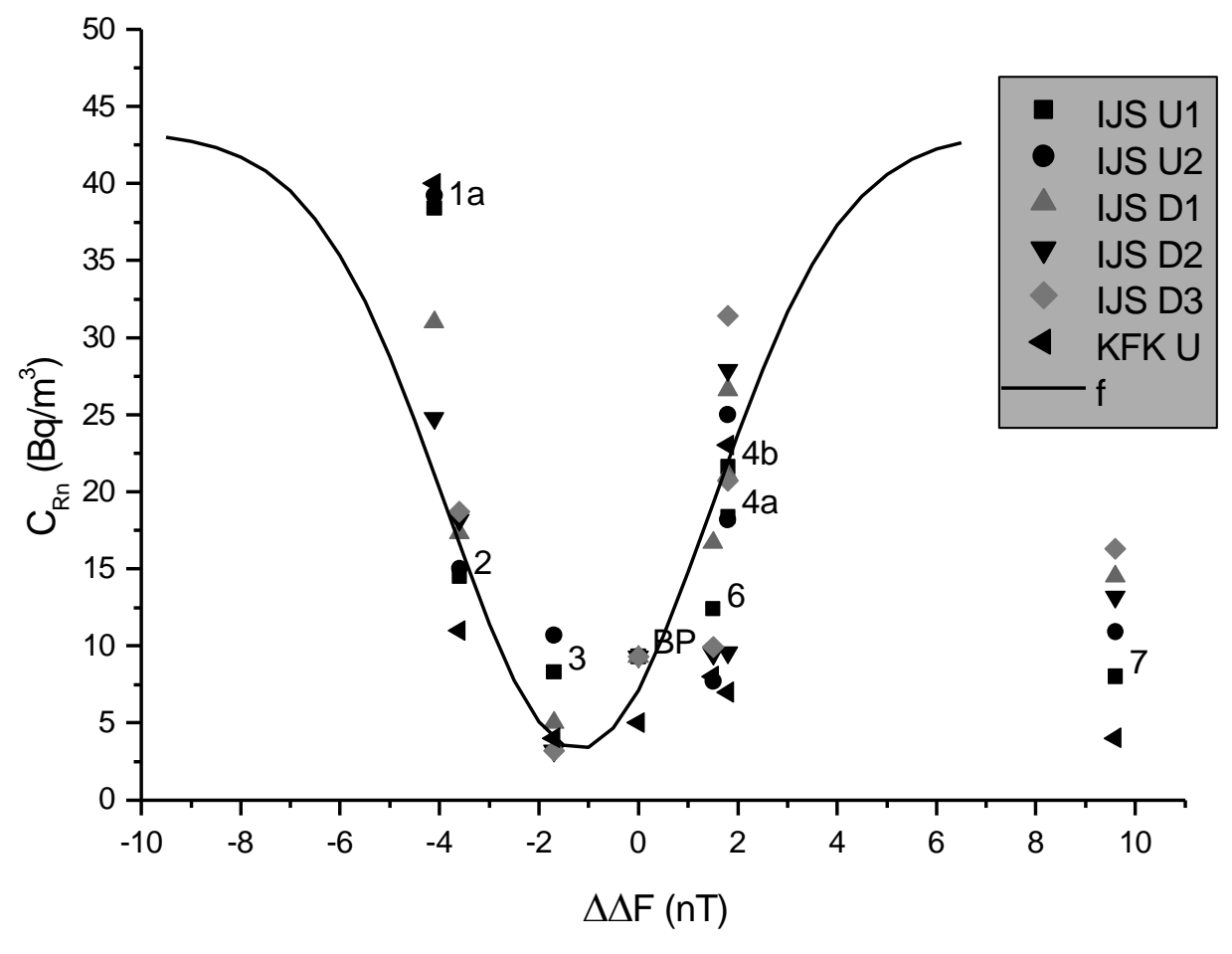

Fig. 4. Annual (2004-05) ${ }^{222} \mathrm{Rn}$ concentration, $\mathrm{C}$, vs change of diference of magnetic field, $\Delta \Delta \mathrm{F}$ in the cycle 200203 at different locations 1a-7 (see Fig. 2). IJS and KFK denote radon dosimeter of J. Stefan Institute, Ljubljana and Nuclear Research Center, Karlsruhe respectively. With D and U radon measurements at the Earth surface and $40 \mathrm{~cm}$ above the surface are denoted. $\mathrm{f}$ is function $\mathrm{C}_{\mathrm{n}}=\mathrm{C}_{\mathrm{BP}}+\mathrm{C}_{\infty}\left[1-\exp \left(-\left|\Delta \Delta \mathrm{F}_{\mathrm{n}}+\Delta \Delta \mathrm{F}_{\mathrm{d}}\right|^{2} / \square \square \Delta \Delta \mathrm{F}_{\mathrm{o}}\right)^{2}\right]$, which was fitted to the experimental data.

Analysis of Figs. 3 and 4 (describing cross-correlation between two precursors - radon and magnetic field variations) shows that the coordinate centre $(0,0,1)$ is situated near the measurement point No. 3 (Fig. 2) corresponding to minimal radon and magnetic field variations. Basing on this analysis and magnetic measurement data (Maksymchuk, 2003), we plotted co-ordinates in Fig. 2. The axis x' coincides with the maximal tangent stress direction in the given region. The axis y' traverses point $(0,0,0.9)$ close to measurement point No. 3 . After that we plot the coordinates of all measurement points on conditional deformation field at $\mathrm{h}=0.9$ (Fig. 5). It is found that the radon concentration ratio for the different pairs of observation points is approximately equal to conditional deformation ratio for corresponding observation points, which is shown in Fig. 5. Hence we may suppose that the spatial distributions of radon concentration and conditional deformation have linear dependence

$$
C_{R n} \sim\left|100 \omega_{x x}\right| .
$$

Note such result is in accordance with predictions of so-called dilatation models (Kasahara, 1981), which well explain observed seismic precursors (in spite of the fact that main of them, i.e. filtration (Scholz et al., 1973) and "dry" (Mjachkin et al., 1975) models are based on the fundamentally different physical processes). 


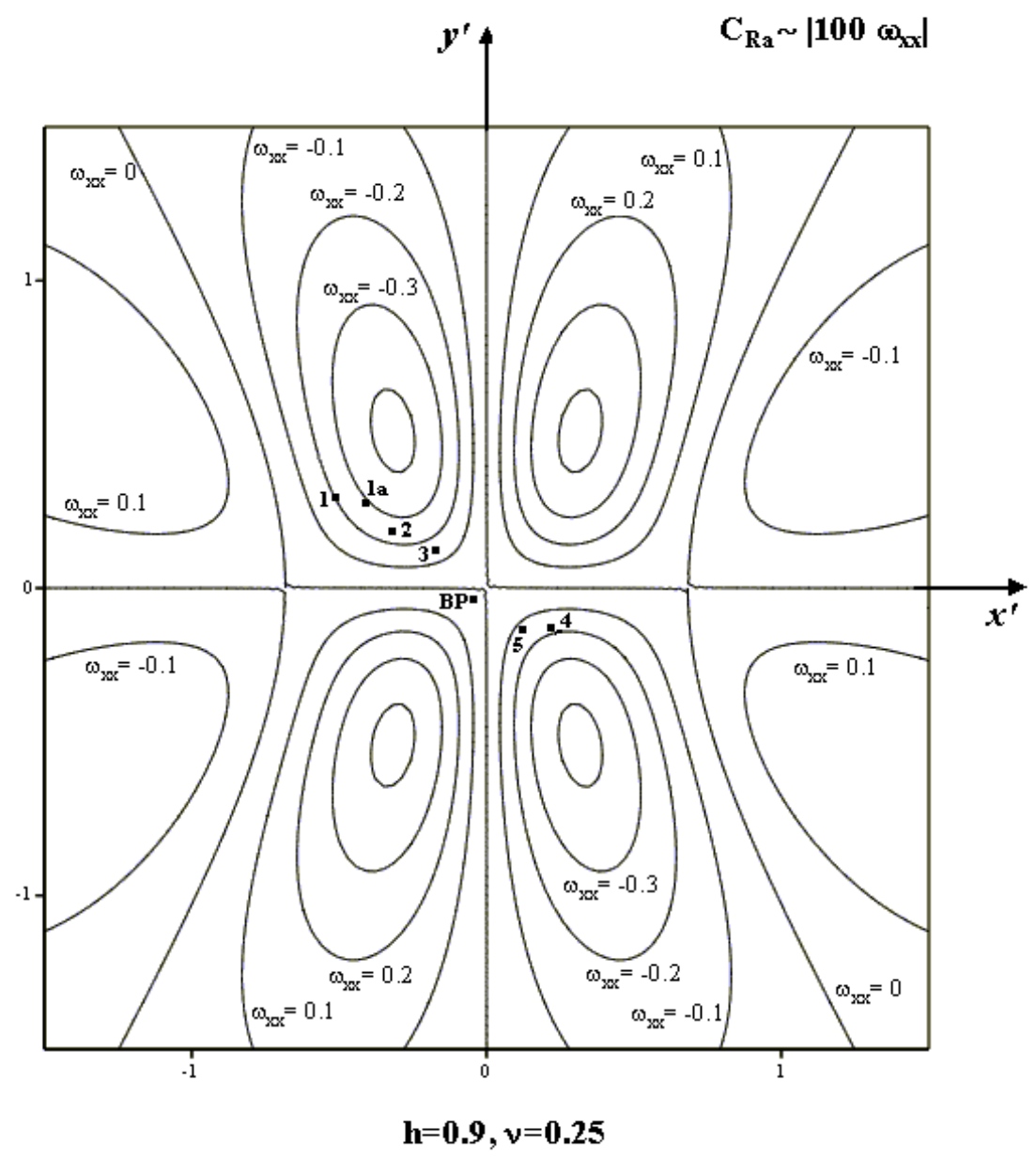

Fig. 5. The distribution of conditional deformation $\omega_{\mathrm{xx}}$ on the Earth's daylight at displacement value of $\mathrm{h}=0.9$. Points 1, 1a, 2, 3, BP, 4, 5 are radon measurement points (see Fig. 2). $\mathrm{C}_{\mathrm{Rn}}, \omega_{\mathrm{xx}}$ and $v$ denote annual radon concentration, conditional deformation and Poissons's ratio, respectively.

This is a very important observation that should be thoroughly tested in further experiments. If this statement is correct, it can become a key moment for the understanding of the physical mechanism of radon emanation at increased tectonic activity, which, as it is well known, can disappears due to slow relaxation processes or fast destruction, i.e. earthquakes (Durrani and Ilić, 1997). In our opinion, one of the convincing methods of the verification of the nonrandom existence of stated physical connection (a linear dependence between spatial radon distribution and conditional deformation) is the simultaneous identification of the points of observation relative to spatial distribution of magnetic field variations.

Since we make comparison with the field observation data in the region of the Western part of the Antarctica Peninsula, let us carry out preliminary analysis of Eq. (35) as applied to this region. Taking into account the distribution of background tectonic stress in this region (Maksymchuk, 2003), we direct the axes $x_{2}$ and $x_{1}$ as it shown in Fig. 2 and axis $\mathrm{x}_{3}-$ to the Earth's center. The absolute value of geomagnetic-field vector in the Western part of the Antarctica Peninsula is about $39500 \mathrm{nT}$, the inclination is $\theta_{1}=-57^{\circ} 14^{\prime}$ and the declination is $\theta_{2}=16^{\circ} 37^{\prime}$.

Let us place the center of area $\mathrm{V}$ on axis $\mathrm{x}_{3}$ as deep as $\mathrm{H}\left(\xi_{1}^{0}=\xi_{2}^{0}=0, \xi_{3}^{0}=\mathrm{H}\right)$ and pass on to dimensionless quantities

$$
\begin{aligned}
& x=x_{1} / \rho, \quad y=x_{2} / \rho, \quad h=H / \rho, \quad r_{d}=r / \rho=\sqrt{x^{2}+y^{2}+(h-0.5)^{2}}, \\
& \beta_{1}=\frac{2}{3} \frac{\rho^{3} \Delta F_{r 1}}{k C V \alpha I \tau}=\frac{1}{2 \pi} \frac{\Delta F_{r 1}}{k C \alpha I \tau} .
\end{aligned}
$$

As a result of this Eq. (35) takes the form

$$
\beta_{1}=-\frac{3}{2 r_{d}^{5}}\left[2\left(b_{1}^{2}+b_{2}^{2}\right) x y+b_{2} b_{3} x(2 h-1)+b_{1} b_{3} y(2 h-1)\right],
$$

where $b_{1}=\sin \left(-57^{\circ} 14^{\prime}\right), b_{2}=\cos 16^{\circ} 37^{\prime}, b_{3}=\sin 16^{\circ} 37^{\prime}$.

The isolines of dimensionless magnetic induction field $\beta_{1}$ at $h=1,2,4$ are presented in Fig. 6. This field has a complex structure dividing into 4 parts by orthogonal zero lines. At first glance, here are possible experimental situations as in the case of deformation or radon measurements (Fig. 2), when the signal is not detected even in immediate proximity to the epicentre of tectonic activity, if observer's location is unsuccessful. But this is not the case. Below we will consider this situation and will show that it is much difficult and interesting in respect to observer. For that we consider the second item $\Delta \mathrm{F}_{\mathrm{r} 2}$ of Eq. (28) 


$$
\Delta F_{r 2}=-\frac{3}{2} k C b_{p} e_{m k p} \nabla_{m} \iiint \frac{e_{i j k} I_{l} s_{i l}^{\prime \prime} R_{j}}{R^{3}} d v .
$$

Eqs. (29) and (43), which describe the first $\Delta \mathrm{F}_{\mathrm{r} 1}$ and the second $\Delta \mathrm{F}_{\mathrm{r} 2}$ components of the total variation of magnetic field $\Delta \mathrm{F}_{\mathrm{r}}$, have distinction of kind. In Eq. (43) the integration is over the all volume of $\mathrm{V}$, therefore at sufficiently large distances from area V, Eq. (29) can be approximated by the field of equivalent magnetic dipole and decreases according to $1 / \mathrm{r}^{3}$ law. As against Eq. (29) the integration in Eq. (43) is over the all volume of regular medium (including the vicinity of the point of observation on the Earth's daylight). The integral in Eq. (43) has dependence like $1 / \mathrm{r}^{2}$ at $\mathrm{R} \rightarrow 0$, hence, it is possible to expect that the main contribution to field $\Delta \mathrm{F}_{\mathrm{r} 2}$ is made by medium volumes in the immediate region of observation point. According to Dobrovolsky (1984), such a property of the future solution makes it possible to reduce Eq. (43) to the following approximation

$$
\Delta F_{r 2} \cong-4 \pi k C I b_{i} b_{l} s_{i l}^{\prime \prime} \text {. }
$$

The independence of $\Delta \mathrm{F}_{\mathrm{r} 2}$ on radius of the distinguished unperturbed medium following from Eq. (44) (Dobrovolsky, 1984) is an important result. Hence, in homogeneous fields of stress, the magnetization $\Delta \mathrm{F}_{\mathrm{r} 2}$ is determined by local effect according to Eq. (44). This means that, when Eq. (44) characterizes the magnetic field variations of unperturbed medium close to observation point, the addition $\Delta \mathrm{F}_{\mathrm{r} 2}$ (specific for given region) may be found by the averaging of the sample of measurements, which made exceptionally on "zero" isolines of the spatial distribution of first component variations $\Delta \mathrm{F}_{\mathrm{r} 1}$. In other words, the observation strategy for observation points location must not only to take into account the real existence of "zero" isolines $\Delta \mathrm{F}_{\mathrm{r} 1}$ but also to use them for the determination of average local addition $\Delta \mathrm{F}_{\mathrm{r} 2}$.

At last, using coordinate system presented in Fig. 2 let us plot coordinates of all measurement points on the field of magnetic induction increment $\Delta \mathrm{F}_{\mathrm{r} 1}$ at $\mathrm{h}=0.9$ (Fig. 7). To ground the choice of value $\mathrm{h}=0.9$ we compared the theoretical values $\Delta \mathrm{F}_{\mathrm{r} 1}$ with the results of field observations in the Western part of the Antarctica Peninsula (Table 2). To obtain the theoretical value of $\Delta \mathrm{F}_{\mathrm{r} 1}$ (Eq. (41)) we used such numerical values of parameters (Dobrovolsky, 1984): $\alpha$ $=0.1, \tau=100 \mathrm{MPa}, \quad \mathrm{k}=10^{-7} \mathrm{mT} \mathrm{A}^{-1}, \mathrm{CI}=10^{-8} \mathrm{~A} \mathrm{~Pa}^{-1} \mathrm{~m}^{-1}$. Then we have

$$
\Delta F_{r 1} \approx 62.8 \beta_{1}, \quad n T \text {. }
$$



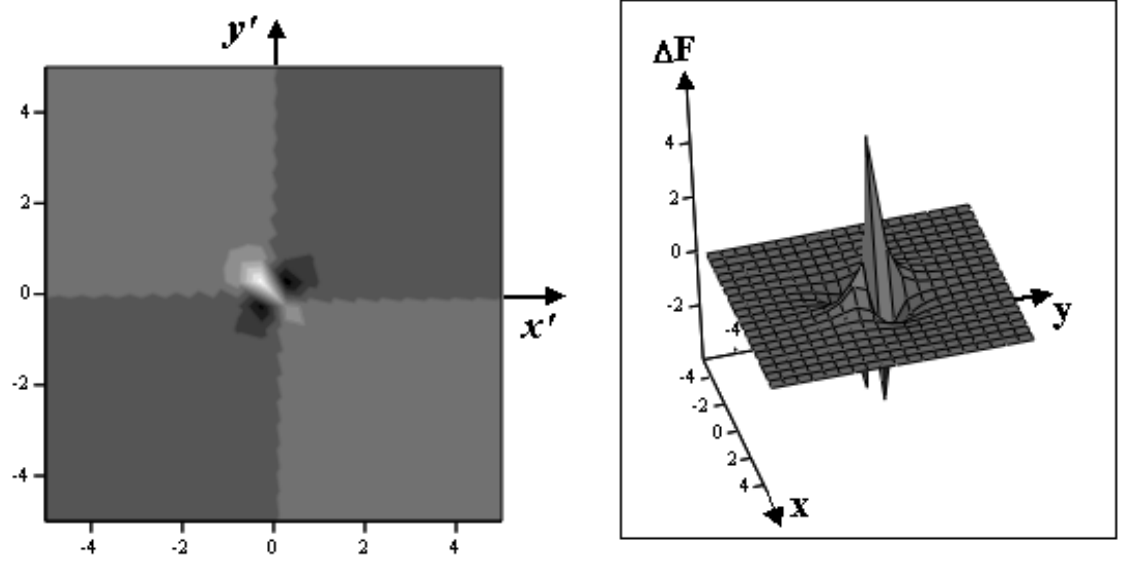

$\mathbf{h}=\mathbf{1}$
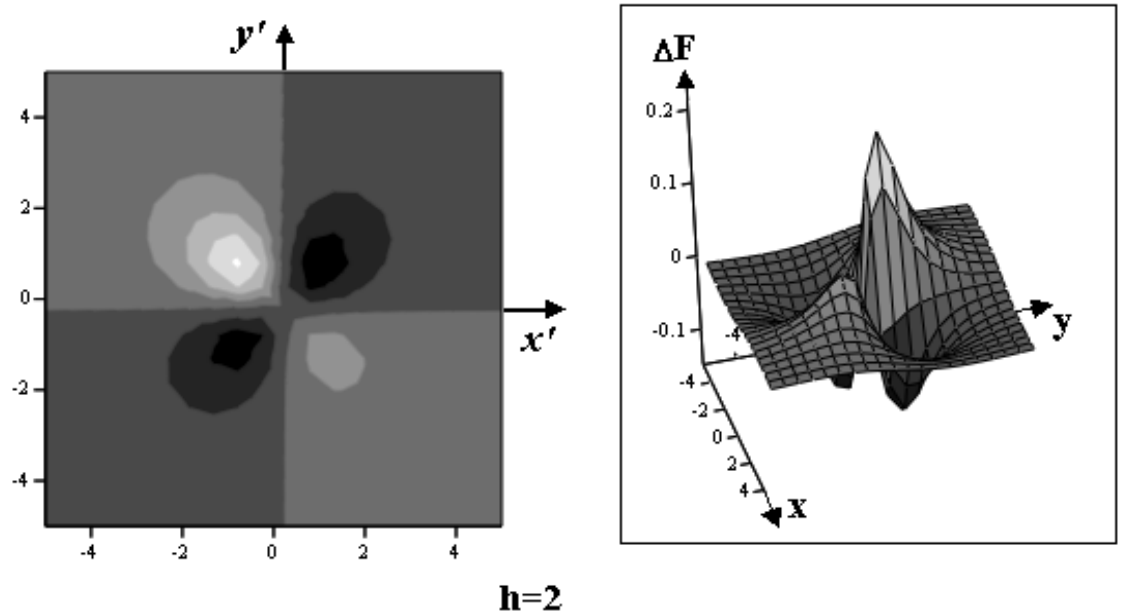

$\mathbf{h}=\mathbf{2}$
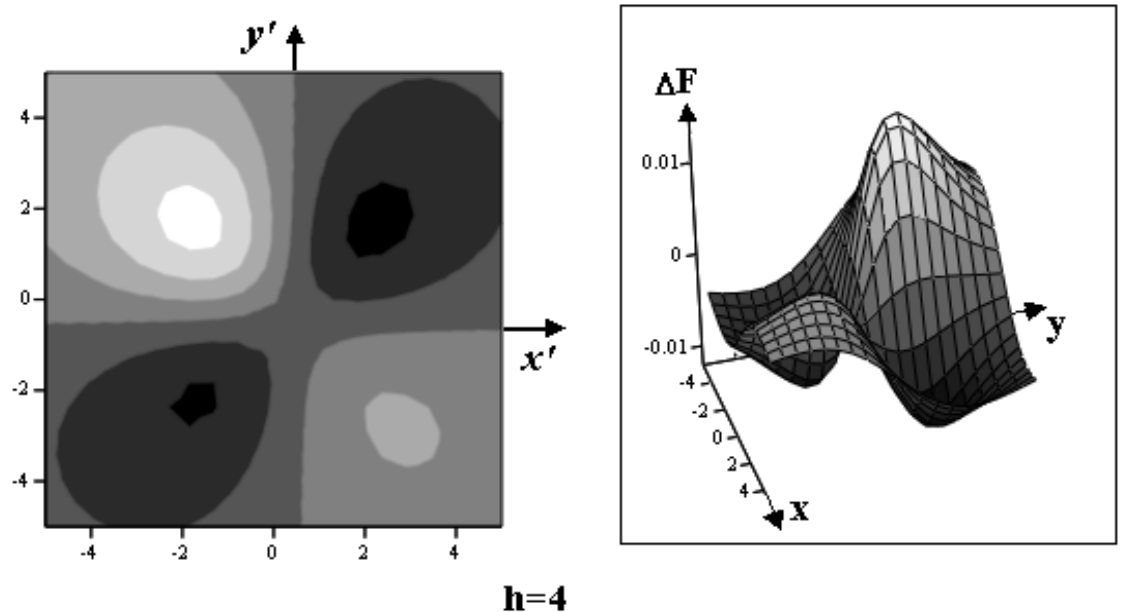

$\mathbf{h}=\mathbf{4}$
Fig. 6. Isolines of

dimensionless

magnetic induction field at different $\mathrm{h}$ on the Earth's daylight.

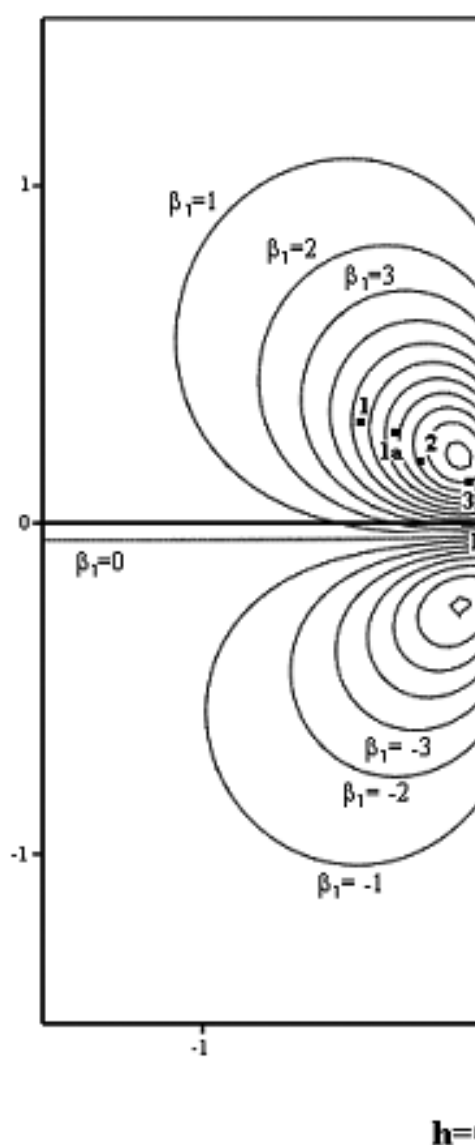

Fig. $\quad$ 7. The distribution of

dimensionless magnetic induction field at displacement value $\mathrm{h}=0.9$. Points $1,1 \mathrm{a}, 2,3, \mathrm{BP}, 4,5$ are the measurement points of magnetic field variations. Symbols $\Delta \mathrm{F}_{\mathrm{r} 1}, \square \beta \square$ and $v \square$ denote the first component of total variation of magnetic field, magnetic induction field and Poison's ratio respectively.

It is obvious that the theoretical value of $\Delta \mathrm{F}_{\mathrm{r} 1}$ (Eq. (45)) is equal to the experimental value (Table 2), when $\beta_{1} \sim 10$. This corresponds to distribution of magnetic induction increment $\Delta \mathrm{F}_{\mathrm{r} 1}$ on the Earth's daylight just at $\mathrm{h}=0.9$ (Fig. 7).

In that case, if to neglect the second component $\Delta \mathrm{F}_{\mathrm{r} 2}$, the ratio of measured variations of magnetic field for the different pairs of observation point (Fig. 2) is approximately equal to the ratio of the theoretical values of magnetic field variations at the corresponding points of observation (Fig. 7). In the same time the experimental value of first components $\Delta \mathrm{F}_{\mathrm{r} 1}$ in point No 2 (Table 2) is substantially smaller then its theoretical value (see Fig. 7). This is explained by the fact that in this case it is not allowed to neglect second component $\Delta \mathrm{F}_{\mathrm{r} 2}$, which (as it was mentioned above) is the characteristic of the piezomagnetic properties of medium in the immediate region of observation point. In other words, the total value of additional magnetic field $\Delta \mathrm{F}_{\mathrm{r} 1}$ in the point No 2 decreases by $\Delta \mathrm{F}_{\mathrm{r} 2}$ due to the strong (anomalous) piezomagnetic effect of medium in the point No 2 (the east part of Barchans Island). 
Results of geomagnetic field and radon concentration measurements at the tectonomagnetic area in the region of the Ukrainian Antarctic Station. The symbols $\Delta F$ and $\Delta \Delta F$ denote the difference of magnetic field at a given and base point for a particular year, and the change in the difference of magnetic field for a given and base point in a measured cycle, respectively. C denotes average annual ${ }^{222} \mathrm{Rn}$ concentration at the earth surface

\begin{tabular}{|c|c|c|c|c|c|c|c|c|c|c|c|c|}
\hline 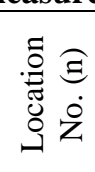 & 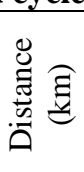 & 灾 & 突 & 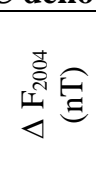 & $\stackrel{\text { 空 }}{\Xi}$ & 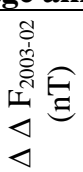 & 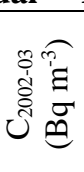 & 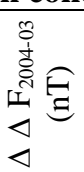 & 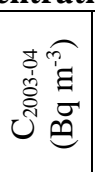 & 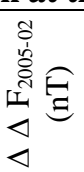 & 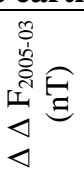 & 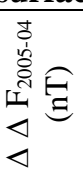 \\
\hline 1 & 0 & 289.2 & 292.1 & 287.3 & 289.4 & 2.9 & & -4.8 & & 0.2 & -2.7 & 2.1 \\
\hline $1 \mathrm{a}$ & 0.3 & 328.6 & 326.7 & 325.7 & 321.6 & -1.9 & & -1 & 42.2 & -7.0 & -5.1 & -4.1 \\
\hline 2 & 0.8 & 125.0 & 127.6 & 126.6 & 123.0 & 2.6 & 8.0 & -1 & 15.8 & -2.0 & -4.6 & -3.6 \\
\hline 3 & 1.7 & 567.7 & 570.9 & 564.6 & 562.9 & 3.2 & 8.7 & -6.3 & 5.7 & -4.8 & -8.0 & -1.7 \\
\hline $3 a$ & 1.9 & 531.2 & 535.4 & 531.5 & 526.6 & 4.2 & & -3.9 & & -4.6 & -8.8 & -4.9 \\
\hline $\mathrm{BP}$ & 2.9 & 0 & 0 & 0 & 0 & 0 & 4.0 & 0 & 8.5 & 0 & 0 & 0 \\
\hline 5 & 3.3 & 40.4 & 41.5 & 36.6 & 38.7 & 1.1 & & -4.9 & & -1.7 & -2.8 & 2.1 \\
\hline 4 & 3.7 & 77.6 & 77.2 & 75.7 & 77.5 & -0.4 & 4.3 & -1.5 & 20.9 & -0.1 & 0.3 & 1.8 \\
\hline 6 & 7.0 & 277.0 & 279.4 & 278.2 & 279.7 & 2.4 & 5.7 & -1.2 & 10.7 & 2.7 & 0.3 & 1.5 \\
\hline 7 & 10.5 & & 1015.9 & 1019.2 & 1028.8 & & & 3.3 & 11.2 & & 12.9 & 9.6 \\
\hline
\end{tabular}

Dynamic changes of magnetic field $\Delta \Delta \mathrm{F}_{2003-02}$ during the period 2002 - 2003 (Table 2) varied from - 1.9 (Barchans Islands) to $+4.2 \mathrm{nT}$ (Three Little Pigs Islands). The definite regularity of their distribution along the profile was noticeable despite the small value of $\Delta \Delta \mathrm{F}$. During the periods 2003-2004 this values was $-4.8 \mathrm{nT}$ on the Barchans Islands, amounts -6.3 $\mathrm{nT}$ on Three Little Pigs Islands to $+3.3 \mathrm{nT}$ (Barchans Islands) and -4. 9 nT (Three Little Pigs Islands) to +6.9 nT (Rasmusen Cape). The repeated observation of 2003, 2004, and 2005 confirms, in general, the stability of $\Delta \Delta \mathrm{F}$ distribution along the profile I - I observed in 2002 (Table 2). Similar anomalous effects, but with a somewhat smaller amplitude, are the general characteristic of active breaks in the Earth's crust and were revealed in many seismoactive zones of the world (Maksymchuk et al., 2002).

So, we may conclude that the selected strategy of measurements (the dashed line of the simultaneous measurements of radon concentration and magnetic field variations in Fig. 2) should lead to the strong cross-correlation between the spatial distributions of the value $\Delta \mathrm{F}_{\mathrm{r}}$ variations (i.e., $\Delta \Delta \mathrm{F}_{\mathrm{r}}$ ) and radon variations in all points of observation (except for points 6 and 7, which are, apparently, the characteristics of totally other physical localization of tectonic activity).

In our opinion, a good coincidence of theoretical and experimental samples of magnetic field variations in this area and simultaneous cross-correlation of the spatial distributions of magnetic field variations $\Delta \Delta \mathrm{F}_{\mathrm{r}}$ and radon variations is a cogent argument in favor of the assumption of linear dependence between spatial distributions of radon and conditional deformation

Finally, the comparative analysis of the spatial distribution of points of observation and their location in Figs. 5 and 7, which describe the variation distribution of radon and magnetic field, shows that the average size $\rho$ of local inhomogeneity along the profile of magnetic measurements approximately is equal to $\rho \sim 4.11 \mathrm{~km}$. We can suppose that this size is comparative to the radius of local inhomogeneity V. If we suppose in turn that the stress of this volume will discharge by destruction i.e. by the earthquake, then, knowing radius $\rho$ of local inhomogeneity $\mathrm{V}$, it is easy to calculate the magnitude $\mathrm{M}$ of potential earthquake (Dambara, 1966)

$$
\lg \rho[\mathrm{km}]=0.5 M-2.27 \Rightarrow M \cong 5.8
$$

and also to estimate the precursor time T (Whitcomb et al., 1973)

$$
\lg T[\text { day }]=0.8 M-1.92 \Rightarrow T=525 \text { days } \text {. }
$$


In order to determine an initial moment of precursor appearance let us plot the time dependences of maximally observed variations of magnetic field (point 3) and radon (point 4), in which the long-term parallel measurements were made. From the dependence shown in Fig. 8 follows that the initial moment of precursor appearance dates from the end of 2003 to beginning of 2004. Within the framework of dilatation models such behavior of precursors (Fig. 8) can be explained by the occurrence of active stage of rock shattering (second stage of seismic cycle in the filtration (Scholz et al., 1973) and "dry" (Mjachkin et al., 1975) models), which can result in reduction of magnetic field $\Delta \mathrm{F}_{\mathrm{r}}$ and the increase of radon emanation, respectively.

Fig. 8. The time dependences of the maximally observed variations of magnetic field $\Delta \mathrm{F}$ (point 3 ) and radon
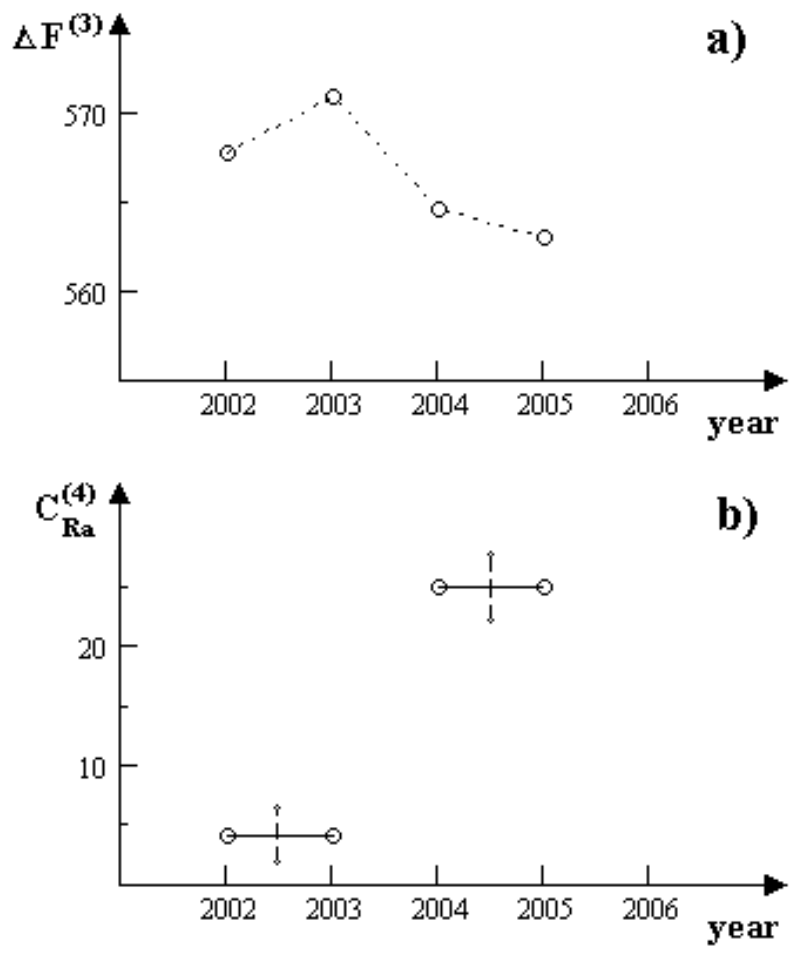

concentration $\mathrm{C}_{\mathrm{Rn}}$ (point 4).

Thus, basing on Eq. (47) we could expect at the end of 2005 in this region the earthquake with epicenter nearby the Three Little Pigs island and magnitude $\mathrm{M}=5.8^{2}$.

Several remarks should be made. First, the stress of disturb area can be discharged not only by the destruction of the Earth crust, but also by the slow mechanisms, for example, by the creep along the breaks. Other mechanisms are also possible, for example, dislocation sliding. If we take into account in addition that the region of Argentine Islands did not reveal seismic activity during the period of observation, then we can conclude that the earthquake in this region has a small probability.

Second, the empirical equations of the type (46), (47) have the statistical character, and we can predict the day of the earthquake on its base only with some probability, if we even know the day of precursors appearance. Moreover, the constants in these equations, which was obtained by statistical averaging of numerous earthquakes, are different in different regions of the Earth, and are unknown for considering region.

\section{Conclusion}

Analysis of the theory and practices of the considered above parallel measurements of the spatial distribution of secondary precursor variations (magnetic field and radon) shows that the problem definition of the estimation of tectonic activity parameters must contain following strategy of measuring and data processing:

1. In order to plot regional coordinates system, which characterizes the area of increased tectonic activity, it is necessary to determine the directions of maximal tangent stresses in given region.

2. To divide the given region into the grid with array pitch about $1-2 \mathrm{~km}$ and to place measuring devices of radon and magnetic field variations in mesh points.

3. Using the symmetry properties of the cross-correlation of magnetic field and radon variations, to determine the center of coordinate system (epicenter of tectonic activity).

4. To determine the absolute value, inclination and declination of magnetic-field vector in the investigated region.

5. To plot the theoretical spatial distribution of deformation and magnetic field variations (first component $\Delta \mathrm{F}_{\mathrm{r} 1}$ ) considering region characteristics at different $(\mathrm{h}=1 \div 5)$ depth of local heterogeneity occurrence (perturbed area $\mathrm{V})$.

\footnotetext{
${ }^{2}$ This paper was finished at the beginning of 2006 and no earthquake was fixed in this region.
} 
6. To plot on a regional coordinate system, whose center and the axes are selected according to item 3 , the coordinates of all measurements of radon and magnetic field variations made according to item 2 .

7. To make the optimal matching of regional coordinate system containing the distribution of experimental measurement point coordinates with the theoretical distribution of deformation (radon) and magnetic field (first component $\Delta \mathrm{F}_{\mathrm{r} 1}$ ) variations plotted according to item 5 .

8. In the case of optimal matching (see item 7) to determine the coordinates and directions of "zero" isolines of the spatial distribution of first component $\Delta \mathrm{F}_{\mathrm{r} 1}$ variations and to make an additional set of measurements of second component $\Delta \mathrm{F}_{\mathrm{r} 2}$.

9. To build the theoretical spatial distributions of total variations of magnetic field when $\Delta \mathrm{F}_{\mathrm{r}}=\Delta \mathrm{F}_{\mathrm{r} 1}+\Delta \mathrm{F}_{\mathrm{r} 2}$ and to make more exact procedure of item 7.

10. Using the procedure of item 7 (specified in item 9) to determine the average radius $\rho$ of local inhomogeneity (perturbed area $\mathrm{V}$ ) and to estimate the magnitude $\mathrm{M}$ by approximate formula (46).

11. For given magnitude $M$ to estimate the precursor time $\mathrm{T}$ of possible earthquake by Eq. (47) or by other known approximate formulas (see, for example, Kasahara's monograph (1981)).

Making all procedures, it is necessary to take into account all remarks described at the end of previous section.

The main conclusion we can make from the above discussion is as follows: studying the tectonic activity of the given region, it is necessary to use the complex approach measuring several known precursors and using some theoretical model. Only such complex approach can give the base to predict possible earthquake in the seismic regions. Concerning the region of Argentine Islands such approach permits to reveal the deformation accumulation in this region which will probably discharge by the slow relaxation mechanisms.

The cause of such accumulation is still unclear, and it should be analysed using all data about the geological structure of the given region. In any case, the revealing of deformation accumulation in the given region should attract the additional attention to this area.

\section{References}

Åkerblom G., Mellander H. 1997. Geology and radon. In: Radon Measurements by Etched Track Detectors: Applications to Radiation Protection, Earth Sciences and the Environment (Eds. S.A. Durrani, R. Ilić), World Scientific, Singapore, pp. 21-49.

Baker P.F. 1982.Cenozoic subduction history of the Pacific margin of the AP: Ridge crest-trench interaction. J. Geol. Soc.London, 139, pp. 787-801.

Balcázar M., 1997. Radon and geothermal energy production. In: Radon Measurements by Etched Track Detectors: Applications to Radiation Protection, Earth Sciences and the Environment (Eds. S.A. Durrani, R. Ilić), World Scientific, Singapore, pp. 345-362.

Breiner S., 1964 Piezomagnetic effects at the time of local earthquakes. Nature, 202, 4934, 790.

Christeson G.L., D. H. N. Baker, J.A. Austin Jr., I.W.D. Dalziel, 2003. Deep crustal structure of Bransfield Strate : initiation of a back ark basin by rift reactivation and prolongation. J. Geophys. Res., 108 (B10), 2492, doi: 10.1029/2003 JB002468.

Dambara T., 1966. Vertical movements of the Earth's crust in relation to the Matsushiro earthquake. J. Geod. Soc. Japan, 12, 18-45 (in Japanese with English abstract).

Dobrovolsky I.P., 1984. Mechanics of tectonic earthquake preparation. Institute of the Earth Physics of Academy of Science USSR, Moscow, 185 pp.

Dobrovolsky I.P., Mjachkin V.I., 1976. The surface displacement of elastic half-space containing an inclusion. In: Seismic Transmission of Nodal Zones. Institute of the Earth Physics of Academy of Science USSR, Moscow, p. 29.

Durrani S.A., Ilić R., Eds., 1997. Radon Measurements by Etched Track Detectors: Applications to Radiation Protection, Earth Sciences and the Environment. World Scientific, Singapore, 416 pp.

Fleischer R.L. 1997. Radon and earthquake prediction. In: Radon Measurements by Etched Track Detectors: Applications to Radiation Protection, Earth Sciences and the Environment (Eds. S.A. Durrani, R. Ilić), World Scientific, Singapore, pp. 285-299.

Guerra M., Lombardi S., 2001. Soil-gas method for tracing neotectonic faults in clay basins: the Pistici field (Southern Italy). Tectonophys. 339, 511-522.

Hakl J., Hunyadi I., Várhegyi A., 1997. Radon monitoring in caves. In: Radon Measurements by Etched Track Detectors: Applications to Radiation Protection, Earth Sciences and the Environment (Eds. S.A. Durrani, R. Ilić), World Scientific, Singapore, pp. 259-283.

Ilić R., Rusov V.D., Pavlovich V.M., Vaschenko V.M., Hanžič L., Bondarchuk Y.A., 2005. Radon in Antarctica. Radiat. Meas. 40, 415-422.

Johnston M.J.S., 1978. Lokal magnetic field variations and stress changes near a slip discontinuity on the San Andreas fault. J. Geomagn. And Geoelect. 30, 511.

Kasahara K, 1981. Earthquake Mechanics. Cambridge University Press, 264 pp.

Khan H.A., Qureshi A.A., Qureshi I.E., 1997. Radon and mineral exploration. In: Radon Measurements by Etched Track Detectors: Applications to Radiation Protection, Earth Sciences and the Environment (Eds. S.A. Durrani, R. Ilić), World Scientific, Singapore, pp. 319-343.

Kharatian K., Travi Y., Igoumnov V., 2002. Radon soil-gas concentrations and local seismicity: case of the Arax basin (Armenia). C.R. Geoscience 334, 179-185.

Majmudar K., 2004. A study of fluctuation in radon concentration behaviour as an aerthquacke presursor. Current Sci. $86,9,1288-1292$. 
Maksymchuk V.Yu., Gorodiskiy Yu.M., Kuznetsova V., 2001. Dynamics of the Anomalous Magnetic Field of Earth, Evrosvit, Lviv, 308 pp (in Ukrainian).

Maksymchuk V.Yu., Kuznetsova V., Chobotok I., Dotsenko I.O., 2002. First results of tectonomagnetic investigations at the Academician Vernadsky Station. Ukrainian Bul. Antarctic Center 4, 197-201.

Maksymchuk V.Yu., Gorodiskiy Yu.M., Chobotok I.O., 2003. The tectonomagnetic researches in the Western Antarctic region. Geodinamics 1, 40-45.

Maksymchuk V.Yu., Chobotok I.O., Romaniuk I.O., 2004. The tectonomagnetic monitoring in the Antarctic station Academic Vernadsky location. In: Proc. $2^{\text {nd }}$ Ukrainian Antarctic Meeting, p. 27.

Maksymchuk V.Yu. et al., 2006. To be published.

Mjachkin V.I., Brace W.F., Sobolev G.A., Dieterich J.H., 1975. Two-models for earthquake forerunners. Pure Appl. Geophys. 113, 168-181.

Monnin M., Seidel L., 1991. Radon and geophysics: recent advances. Nucl. Tracks Radiat. Meas. 19, 1-4, 375-382.

Monnin M.M., Seidel J.L., 1997. Radon and volcanic surveillance. In: Radon Measurements by Etched Track Detectors: Applications to Radiation Protection, Earth Sciences and the Environment (Eds. S.A. Durrani, R. Ilić), World Scientific, Singapore, pp. 301-318.

Novatsky V., 1975. The Theory of Elasticity. Mir, Moscow, 872 pp.

Rusov V.D., Pavlovich V.N, Ilić, Jaćimović R., Bondarchuk Y.A, Vaschenko V. N., Mihalus, O.T.2005. On the fractal mechanism of interrelation between the genesis, size and content of atmospheric aerosols in different regions of the Earth. Atmosph. Environm., submited for publication.

Rusov V.D., Glushkov V.D., Vaschenko V.M., Kocenko S.I., Tarasov V.A., Zelentsova T.N. Saranjuk D., Shevcenko V., 2006. Self organization of the criticality of $1 / \mathrm{f}^{\square} \square$ noice of statistics of registration of surface radon as dynamic indicator of earthquackes: theory and experiments. To be submitted for publication in Phys. Rev. E.

Scholz C.H., Sykes L.R., Aggarwal Y.P., 1973. Earthquake prediction: a physical basis. Science 181, 803.

Segovia N., 1991. Radon and volcanic activity: resent advance. Nucl. Tracks Rad. Meas. 19, 1-4, 409-413.

Singh M., Ramola R.C., Singh B., Singh S., Virk H.S., 1991. Subsurface soil gas radon changes associated with earthquakes. Nucl. Tracks Radiat. Meas. 19, 1-4, 417-420.

Skovorodkin Yu.P., 1985. Study of Tectonic Processes with Magnetometrical Methods. Moscow Institute of Earth Physics, Academy of Science of USSR, Moscow, 197 pp (in Russian).

Skvarč J., Golovchenko A.N., 2001. A method of trajectory tracing of Z $\leq 10$ ions in the energy region below 300 MeV/u. Radiat. Meas. 34, 1-6, 113-118.

Smellie J. L., Recent observations on the volcanic history of Deception Island, South Shetland Islands, Br. Antarct. Surv. Sci. Rep., 81, 83-85, 1988.

Stacey F.D., 1972. Theory of the piezomagnetic effect in titanomagnetite bearing rocks. Pageoph 97, 146.

Šutej T., R. Ilić, R., Najžer, M., 1986. Response of track-etch dosemeters to environmental radon. Nucl. Tracks Radiat. Meas. 15, 1-4, 547-550.

Urban M., Piesch E.A., 1981. Low level environmental radon dosimetry with a passive track etch detector device. Radiat. Prot. Dosim. 1, 2, 97-109.

Whitcomb J.H., Garmany J.D., Anderson D.L., 1973. Earthquake prediction: variation of seismic velocities before the San Fernando earthquake. Science 180, 632-635.

Zmazek B., Todorovski L., Džerovski S., Vaupotič J., Kobal I., 2003. Application of decision trees to the analysis of soil radon data for earthquacke prediction. Appl. Radiat. Isot. 58, 697-706. 